\title{
NTF3 Is a Novel Target Gene of the Transcription Factor POU3F2 and Is Required for Neuronal Differentiation
}

\author{
Yi-Mei J. Lin ${ }^{1} \cdot$ I-Lun Hsin ${ }^{2} \cdot$ H. Sunny Sun ${ }^{3} \cdot$ Shankung Lin ${ }^{2} \cdot$ Yen-Ling Lai ${ }^{2} \cdot$ Hsuan-Ying Chen ${ }^{2} \cdot$ Ting-Yu Chen $^{2}$. \\ Ya-Ping Chen ${ }^{2} \cdot$ Yi-Ting Shen ${ }^{1} \cdot$ Hung-Ming $\mathrm{Wu}^{2,4,5}$
}

Received: 24 June 2017 / Accepted: 7 March 2018 / Published online: 16 March 2018

(C) The Author(s) 2018

\begin{abstract}
POU-homeodomain transcription factor POU3F2 is a critical transcription factor that participates in neuronal differentiation. However, little is known about its downstream mediators. Here genome-wide analyses of a human neuronal differentiation cell model, NT2D1, suggested neurotrophin-3 (NTF3), a key mediator of neuronal development during the early neurogenic period, as a putative regulatory target of POU3F2. Western blot, cDNA microarray, and real-time quantitative PCR analyses showed that POU3F2 and NTF3 were upregulated during neuronal differentiation. Next-generation-sequence-based POU3F2 chromatin immunoprecipitation-sequencing and genome-wide in silico prediction demonstrated that POU3F2 binds to the NTF3 promoter during neuronal differentiation. Furthermore, unidirectional deletion or mutation of the binding site of POU3F2 in the NTF3 promoter decreased promoter-driven luciferase activity, indicating that POU $3 \mathrm{~F} 2$ is a positive regulator of NTF3 promoter activity. While NTF3 knockdown resulted in decreased viability and differentiation of NT2D1 cells, and POU3F2 knockdown downregulated NTF3 expression, recombinant NTF3 significantly rescued viable neuronal cells from NTF3- or POU3F2-knockdown cell cultures. Moreover, immunostaining showed colocalization of POU3F2 and NTF3 in developing mouse neurons. Thus, our data suggest that NTF3 is a novel target gene of POU3F2 and that the POU3F2/NTF3 pathway plays a role in the process of neuronal differentiation.
\end{abstract}

Keywords POU3F2 $\cdot$ Neuron differentiation $\cdot$ Neurotrophin 3

\section{Introduction}

POU-homeodomain transcription factor POU3F2 (also known as BRN2) is one of four closely related Class III

Electronic supplementary material The online version of this article (https://doi.org/10.1007/s12035-018-0995-y) contains supplementary material, which is available to authorized users.

Hung-Ming Wu

18288@cch.org.tw

1 Institute of Biomedical sciences, National Chung Hsing University, Taichung City, Taiwan

2 Inflammation Research \& Drug Development Center, Changhua Christian Hospital, No. 135 Nanxiao Street, Changhua 500, Taiwan

3 Institute of Molecular Medicine, National Cheng Kung University, Tainan, Taiwan

4 Graduate Institute of Acupuncture Science, China Medical University, Taichung, Taiwan

5 Department of Neurology, Changhua Christian Hospital, Changhua, Taiwan
POU proteins that are predominantly expressed in the central nervous system (CNS) and are involved in patterning the embryonic brain $[1,2]$. POU3F2 was originally found in neuronal cells but has also been detected in oligodendrocytes and melanocytes [2-4]. This brain-specific transcription factor is upregulated in the progenitor cells in the subventricular zone, intermediate zone, and outer layer of the neocortex during the early stages of embryonic brain development $[5,6]$, when POU3F2 contributes to neural formation and cell fate determination [7] and also regulates cortical neural migration [8], neurogenesis, and positioning of cortical neurons $[9,10]$. Targeted inactivation or deletion of the Pou $3 f 2$ gene in mice results in complete loss of the development of specific neuronal lineages in paraventricular nuclei and supraoptic nuclei in the hypothalamus [2]. Forced expression of POU3F2 and other neuronal transcription factors (ASCL1, MYT1L, and NEUROD1) has been shown to induce neuronal fate in pluripotent stem cells and to convert fetal and postnatal human fibroblasts or somatic cells into functional neurons [11-15]. More recently, Urban and colleagues demonstrated that POU3F2 was essential for retinoic acid-induced neuronal 
differentiation from mouse embryonic stem cells by regulating a set of target genes via binding to the genomic locus Zic 1 [16]. These results indicate that POU3F2 is a critical transcription factor in the conversion of embryonic stem cells into neurons and glial cells; however, little is known about the neurogenic genes it regulates.

Neurotrophin-3 (NTF3), a member of the neurotrophin family that includes nerve growth factor, brain-derived neurotrophic factors, and neurotrophin $4 / 5$, has emerged as a key mediator of neuronal development during the early neurogenic period as well as throughout adulthood. NTF3 has been shown to maintain synapse function and synaptogenesis [17, 18]. Neurotrophins bind selectively to specific tyrosine receptor kinases to relay signals in various signaling pathways [19]. The binding of NTF3 to TrkC induces PI3K/Akt and RAS/ ERK signaling pathways that regulate cell growth, survival, and differentiation $[19,20]$. Functional deficiency in NTF3 has been shown to cause severe neuronal deficits and early postnatal death in mice [21]. Furthermore, motor neurons derived from conditional Ntf3-knockout embryos showed increased apoptosis and abnormal projection of the centralbranch-innervating motor neuron, suggesting that NTF3 is critically important for survival and axonal projection of neurons [22].

In this study, we applied a genome-wide integrated analysis of the human NT2D1 cell model to identify putative POU3F2 target genes. When properly induced, NT2D1 cells are capable of differentiation into neuronal cells [23]. Our strategy, which combined computational-based and next-generationsequencing-based genome-wide POU3F2-binding-site screening (ChIP-seq) to investigate the neuronal differentiation process, successfully identified several hundred putative genes that are targeted by POU3F2 during neuronal differentiation. One of the putative genes was NTF3. Accordingly, we have investigated whether POU3F2 regulated NTF3 expression and the role of the POU3F2/NTF3 pathway in the early stages of neuronal development using a human pluripotent embryonic carcinoma cell line NTERA2 cl.D1 (NT2D1). Our data show that NTF3 is a novel target gene of POU3F2 and provide new insight into the mechanisms through which POU3F2/NTF3 regulates neuronal differentiation.

\section{Materials and Methods}

Animals Timed-pregnant adult $\mathrm{C} 57 \mathrm{BL} / 6 \mathrm{~J}$ female mice were purchased from the National Lab Animal Center (Taiwan). All procedures were approved by the Animal Care and Use Committee of Changhua Christian Hospital.

Primary Neuron-Enriched Cultures Mouse primary neuronenriched cultures were prepared using a previously described protocol [24]. Briefly, cerebral tissues were dissected from mouse embryonic day (E) 14.5. Cells were dissociated by gentle mechanical trituration and immediately seeded at a density of $5 \times 10^{5}$ cells/well in 24 -well plates pre-coated with poly D-lysine $(20 \mu \mathrm{g} / \mathrm{ml})$. Plates were maintained at $37{ }^{\circ} \mathrm{C}$ in a humidified atmosphere of $5 \% \mathrm{CO}_{2}$ and $95 \%$ air. Forty-eight hours after seeding, cytosine $\beta$-D-arabinofuranoside $(10 \mu \mathrm{M})$ was added to prevent glial proliferation and was then removed $24 \mathrm{~h}$ later. The cultures were subsequently maintained in serum-free neurobasal medium (Invitrogen, Carlsbad, CA, USA) supplemented with $2 \%$ B27 and $2 \mathrm{mM}$ glutamine and were given fresh medium every 3-4 days. Neuron-enriched cultures were $98 \%$ pure, and microglia-depleted cultures were $95 \%$ pure.

NTERA2 cl.D1 Cell Line Human pluripotent embryonic carcinoma NTERA2 cl.D1 (NT2D1) cells (ATCC, CRL1973) were cultured in Dulbecco's Modified Eagle's Medium (DMEM) supplemented with 10\% fetal bovine serum (FBS). The cells were incubated at $37^{\circ} \mathrm{C}$ in a humidified atmosphere containing $5 \% \mathrm{CO}_{2}$ and used over a restricted culture period of 10 passages.

Neuronal Differentiation of NT2D1 Cells Differentiation of NT2D1 cells was conducted as described previously [23, 25, 26]. Briefly, confluent NT2D1 were first cultured in preinduction DMEM medium supplemented with 20\% FBS and $10 \mathrm{ng} / \mathrm{ml} \mathrm{bFGF}$ (Gibco, 13256-029) for $24 \mathrm{~h}$. Cells were then incubated in neuron induction medium (GlutaMax DMEM, $10 \mathrm{ng} / \mathrm{ml}$ bFGF, $10 \mathrm{ng} / \mathrm{ml}$ PDGF-BB, $100 \mu \mathrm{M}$ BHA, $10 \mu \mathrm{M}$ forskolin, $2 \mathrm{mM}$ valproic acid, $25 \mathrm{mM} \mathrm{KCl}, 2 \% \mathrm{DMSO}$, and $1 \times$ B27 supplement) for another $24 \mathrm{~h}$ to induce neuronal differentiation. For the NTF3 treatment experiment, NTF3 recombinant protein (ProSpec, CYT-257) was added to the neuron induction medium during the neuron differentiation step.

\section{Genome-wide In Silico Prediction of Putative POU3F2 Binding} Sites and Bioinformatic Promoter Analysis of NTF3 Promoter Sequences We applied computer-based search tools to search for potential target genes of POU3F2. One was "The Binding Element Searching Tools (The BEST; NCKU Bioinformatics Center)," which was used to build a customized hidden Markov model (HMM) based on a set of sequences containing known POU3F2 binding sites. This model was then used to screen the promoter regions of all known human genes. The other one was the "AnGEL genome-wide CRM Searches" in the Transcription Element Search System (TESS) web server, which was used to scan the promoter sequences of all identified human genes for the presence of the conserved POU3F2 recognition site. By combining the results from these two bioinformatic tools, we obtained a set of putative target genes of POU3F2. To study the transcriptional regulation of the NTF3 gene, the region ranging from $\mathrm{nt}-2000$ to $\mathrm{nt} 221$ from the transcription start 
site of the $N T F 3$ gene was used to predict the potential transcription factors binding elements. The sequences upstream of the human NTF3 gene (NC_000006.11) were obtained from the National Center for Biotechnology Information (NCBI) and screened using TESS software (http://www. cbil.upenn.edu/cgi-bin/tess/tess).

DNA Affinity Precipitation Assay To detect the interaction between the POU3F2 protein and Ntf3 gene, a DNA affinity precipitation assay (DAPA) was performed. Briefly, double-stranded, 5'-end-biotinylated oligonucleotides prepared by PCR were incubated with nuclear extracts from NT2D1 cells in a $100-\mu 1$ reaction mixture $(20 \mathrm{mM}$ sodium phosphate, $\mathrm{pH} 8.0,100 \mathrm{mM} \mathrm{NaCl}, 7 \%$ glycerol, $0.5 \mathrm{mM}$ EDTA, $2 \mathrm{mM}$ dithiothreitol, $0.1 \mathrm{mg} / \mathrm{ml} \mathrm{BSA}, 0.02 \%$ Tween, and $0.02 \mathrm{mg} / \mathrm{ml}$ poly (dI-dC)) at $4{ }^{\circ} \mathrm{C}$ for $16 \mathrm{~h}$. The mixtures were then incubated with $0.74 \%$ for an additional $30 \mathrm{~min}$ on ice to cross-link the DNA-protein complexes. The cross-linked complexes were pulled down by using M-280 Streptavidin Dynabeads (Dynal Biotech ASA, Oslo, Norway) following the manufacturer's instructions and analyzed by Western blotting using antiPOU3F2 antibody (Abcam, Cambridge, UK). The DNA sequences for DAPA were as follows: AADC: 5'-GCTG CTCAGTAAATAATGCAGAGC-3', and 5'-GCTCTGCAT TAT T TAC T GA GCA GC-3'; NTF 3: $5^{\prime}$ - T T TC AAGGTATTTGGATTTTTTGAAC-C-3' and 5'-GGTT CAAAAAATCCAAATACCTTGAAA-3'; NTF3_Mutant: 5'-GG-TTCAAGCGCCCGCGCACCTTGAAA-3' and 5'TTTCAAGGTGCGCGGGCGC-TTGTAACC-3'.

\section{Next-Generation-Sequence-Based POU3F2 ChIP-Sequencing} POU3F2 chromatin immunoprecipitation (ChIP) samples were prepared from NT2D1 cells harvested at the indicated times after neurogenic induction. These NT2D1 cells were fixed with $1 \%$ formaldehyde. Nuclear lysates were sonicated with a sonicator to shear the chromatin into fragments of approximately $200 \mathrm{bp}-1 \mathrm{~kb}$ in size. The sonicated lysates were then incubated with anti-POU3F2 antibody (sc-6029, Santa Cruz Biotechnology, Inc., Dallas, TX) and the protein-DNA complexes precipitated with protein $\mathrm{A} / \mathrm{G}$ agarose beads. After washing and reversal of the cross-link, DNA was recovered by phenol/chloroform extraction and sequenced simultaneously using the Genome Analyzer and Solexa ${ }^{\circledR}$ Sequencing technology (Illumina, Inc., San Diego, CA).

\section{Promoter Assay Construct and Plasmid DNA Extraction} Different lengths of $N T F 3$ promoter were cloned upstream of the firefly luciferase reporter separately in the pGL3Basic vector. The NTF3 mut pGL3-b plasmid with a mutated POU3F2 binding site (5'-ATTTTGGATT-3' to 5'-ATGG GGAGG-3') was provided by Protech Technology Enterprise Co, Ltd (Taipei, Taiwan).
Plasmid Transfection and Luciferase Assay NT2D1 cells were seeded onto 24 -well culture dishes for $24 \mathrm{~h}$ and co-transfected with pGL3-basic promoter-driven Firefly luciferase reporter plasmid and Simian Virus 40 promoter-driven Renilla (pRLSV40) plasmid using jetPEI (101-10, Polyplus Transfection, France) and incubated for $24 \mathrm{~h}$. The activities of Firefly luciferase and Renilla luciferase were measured by using the DualLuciferase Reporter Assay System (Promega, Wisconsin, USA) and a GloMax ${ }^{\text {TM }}$ 20/20 Luminometer. The transfection experiments and luciferase assay were performed according to the manufacturer's instructions.

VSV-G Pseudotyped Lentivirus-shRNA Production and Infection The plasmids pshRNA ${ } O U 3 F 2$ (clone ID:TRCN0000230048), pshRNA ${ }_{N T F 3}$ (clone ID:TRCN0000378856), and pshRNA $L u c$ (clone ID:TRCN0000231693) harboring shRNA targeting POU 3F2, NTF3, and luciferase mRNAs, respectively, were purchased from the National RNAi Core Facility at Academia Sinica, Taiwan. Virus preparation and cell infection were performed as suggested by the plasmids' provider (http://rnai. genmed.sinica.edu.tw/) and in previous studies [27, 28].

Whole Cell Lysate Extraction and Immunoblotting For whole cell lysate extraction, NT2D1 cells were lysed in RIPA buffer (50 mM Tris-HCl pH 7.5, $150 \mathrm{mM} \mathrm{NaCl}, 0.1 \%$ SDS, $0.5 \%$ sodium deoxycholate, $1 \%$ NP- $40,1 \times$ protease inhibitor cocktail) for $10 \mathrm{~min}$ on ice and then centrifuged at $16,000 \times \mathrm{g}$ for $15 \mathrm{~min}$ to remove debris. Total proteins from each sample were fractionated by SDS-PAGE and then transferred to a polyvinylidene difluoride (PVDF) membrane. Monoclonal antibodies that recognize POU3F2 (\#12137, Cell signaling), POU3F3 (ab90727, Abcam), NTF3 (ab65804, Abcam), ß3Tubulin (ab18207, Abcam), and GAPDH (NB300-221, NOVUS) were used to detect the expressions of POU3F2, POU3F3, NTF3, $\beta 3$-Tubulin, and GAPDH, respectively. Membranes were washed for $10 \mathrm{~min}$ in $0.1 \%$ TBS-Tween 20 , incubated in HRP-conjugated secondary antibody for $1 \mathrm{~h}$, washed again, and then analyzed with the GeneGnome chemiluminescence imaging system.

Chromatin Immunoprecipitation and Polymerase Chain Reaction For ChIP, formaldehyde cross-linked cells were sonicated for 40 cycles (30 s on/30 s off) using a Misonix Q700 Sonicator (Qsonica, Newtown, CT) at $20 \%$ power amplitude. Briefly, $5 \mu \mathrm{g}$ POU3F2 antibody and naive $\mathrm{IgG}$ were mixed with Dynabeads protein-G beads at $4{ }^{\circ} \mathrm{C}$ for $2 \mathrm{~h}$. After the bead-antibody interaction, the antibody-conjugated beads were used to pull down the POU3F2-bound DNA fragments. Cross-links were reversed by incubation at $65{ }^{\circ} \mathrm{C}$ overnight. The proteins were digested by $20 \mu \mathrm{g}$ proteinase $\mathrm{K}$ at $50^{\circ} \mathrm{C}$ for $2 \mathrm{~h}$. The DNA fragments were purified by phenol/chloroform precipitation. The DNA sequences of the NTF3 promoter 
occupied by POU3F2 were amplified by polymerase chain reaction (PCR). The primers used were forward 5 -GTGG GTGCAGTTCCGATG-3' and reverse 5'-CGCT CCTCACATCATCTCCT-3'.

Immunofluorescence POU3F2-knockdown and control NT2D1 cells were seeded onto coverslips in six-well plates at a density of $8 \times 10^{4}$ cells/well for the neuronal immunofluorescence assay. After exposure to neural induction medium at the indicated time points, the cells were washed in phosphatebuffered saline (PBS), fixed in 4\% paraformaldehyde in PBS for $10 \mathrm{~min}$ at room temperature, permeabilized with $1 \%$ Triton X-100 in PBS for 10 min, and then blocked in 1\% BSA in PBST for $1 \mathrm{~h}$. Subsequently, cells were hybridized with anti- $\beta 3$-Tubulin (ab18207, Abcam) and anti-phospho-TrkC (\#NBP1-03448, Novus) antibodies overnight in blocking buffer at $4{ }^{\circ} \mathrm{C}$. Cells were also exposed to donkey antirabbit-FITC antibody at room temperature for $1 \mathrm{~h}$. The cells were stained with 4', 6-diamidino-2-phenylindole (DAPI) for $5 \mathrm{~min}$ at room temperature and observed using a fluorescence microscope.

Mouse primary cultured neurons in a 24-well plate were fixed in $4 \%$ paraformaldehyde for $10 \mathrm{~min}$. Mouse brains harvested at E18.5 or $\mathrm{P} 0$ were dissected and post-fixed overnight in $4 \%$ paraformaldehyde at $4{ }^{\circ} \mathrm{C}$ for $16 \mathrm{~h}$. Coronal frozen sections $(20 \mu \mathrm{m}$ thick) were prepared. Primary cultured neurons and brain sections were permeabilized with $1 \%$ Triton X100 in PBS for 10 min and then blocked in $1 \%$ bovine serum albumin in PBST for $1 \mathrm{~h}$. Then, primary cultured neurons and slices were incubated overnight at $4{ }^{\circ} \mathrm{C}$ in blocking buffer containing goat anti-NTF3 antibody (\#NBP1-46517, Novus) and rabbit anti-POU3F2 antibody (\#12137S, Cell Signaling).

A

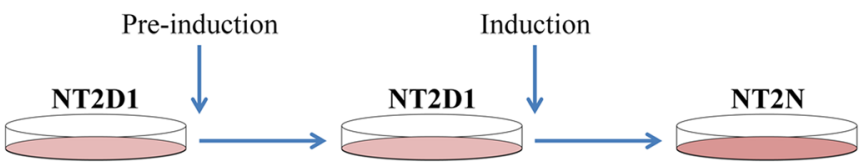

B
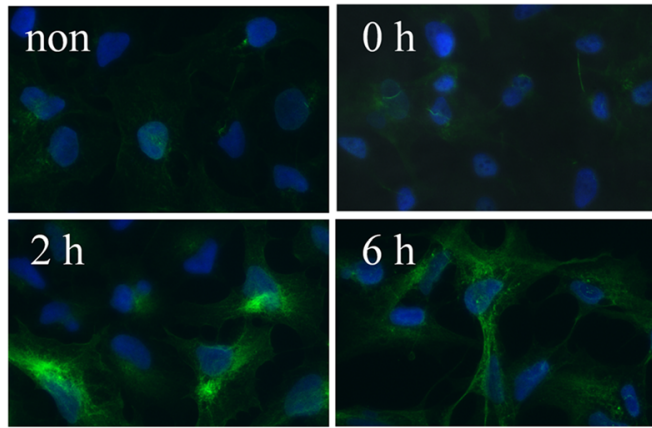

C

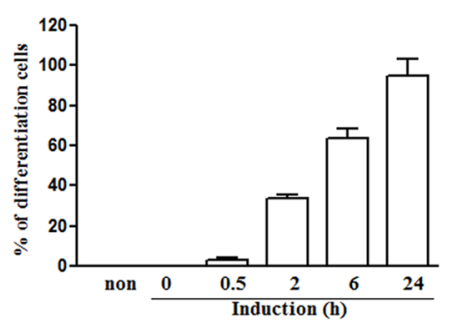

Fig. 1 Upregulation of the transcription factors POU3F2 and NTF3 during neuronal differentiation of NT2D1. a The protocol for neuronal induction of NT2D1 cells is schematized. b $\beta 3$-tubulin staining for neuronal cells in NT2D1 cells untreated (non) and treated with neuronal induction medium at the indicated time points. c Quantification of $\beta 3$ tubulin-positive cells. d Immunoblotting analysis for POU3F2, POU3F3, $\beta 3$-tubulin, and NTF3 in NT2D1 cells untreated or treated with neuronal induction medium at the indicated time points. The values show the expression relative to that of untreated cells (to which a value of 1 was
E

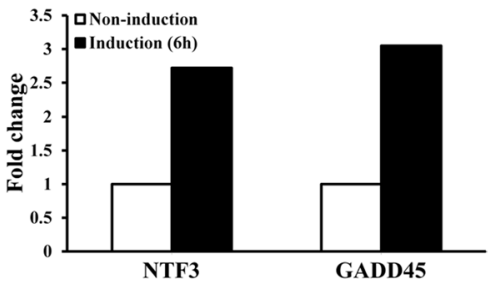

F

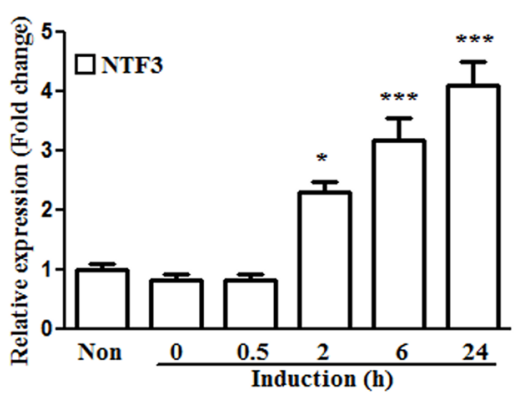

G
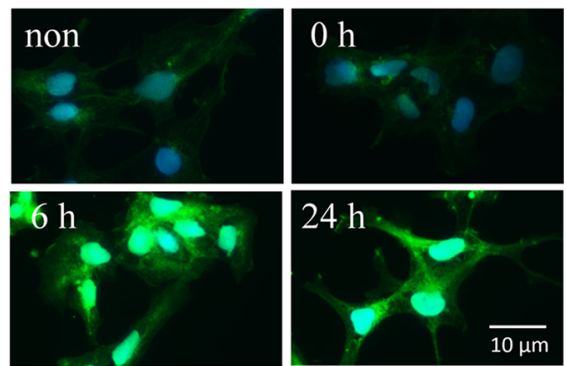

assigned). e Microarray analysis showed that neuronal induction for $6 \mathrm{~h}$ increased the expression of NTF3 and GADD45 in NT2D1 cells. $\mathbf{f}$ NFF mRNA expression after neuronal induction was analyzed by real-time PCR. The levels of mRNA were calculated as the relative expression compared with that of non-induced NT2D1 cells. GAPDH mRNA was used as a control. $* p<0.05 ; * * * p<0.001$. g Phospho-TrkC (Tyr820) staining in treated and untreated NT2D1 cells. Values are presented as mean \pm SEM of three independent experiments for $\mathbf{c}$ and $\mathbf{f}$ 
A

-1823 CAGATGAGAGGGAGAAAAGCAGAACCCGACAGAGCACGCCCAATCCAAACCTTCATGGTG -1763 CTGTGTGGCTGGGTGGAGGGAACGACTCGGCAGCCTCTTCTGGCCCTGAGGAAGACGTCG

-1703 ATATTTTGGCACGAGGGGAGCCACTGAAGGACTACCCTACCCTTGCGAGGGACCGCAGGA -1643 GGTGACGCCCCTGGGCCTCGGTGGGCGCTTCTGGCGGTTTTCGATGTGGCAACCCCCATC -1583 AGCCAGGATAATGATGAGGCAGGTACAATCTATCTAGTACGCAGCCCCCAGACTCCCCCC -1523 TCCCTTCCCACCTCCCCATCCAACCCCCCAGCTACTCTCTGCGGCCGGTTGGTCCTGAAC POU3F2
-1463 TGGTGGGTGCAGTTCCGATGTTTAACCAAATTCTCAAGCAATTTCAAGGTATTTGATT -1403 TTTGAACCTGCCCCCTABCCG

-1403 TTTGAACCTGGGCCCTAACCGAAACGCGGAACGGCTTGCTTATTAGACACCTCGAACGAC -1343 AGGGCAGGGAGGAAACGGGATACTCGCTGCCCTTCCCAGTCGCGCGTGAGTCAAAAGGTC -1283 CTGGCAGGAGATGATGTGAGGAGGGCTGAAGTGGCAG . . . . . . . . .

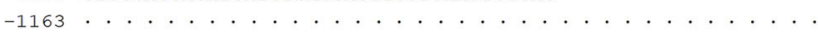

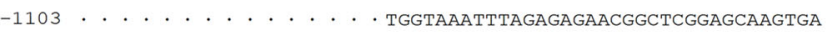

1043 GGTCTAACGGGCAGCTAAAATTATCTCCAAATAAGAGATTTTGACCCCCTCCCCCTATCC -983 TCTCCTCGAATGTATCCACCGGTGGGGAAGTGAGCGTCATTACTTTCGGGGCGCCACGAA -923 CAGGTTTGTTTGTTGCTCGCCTTTCCTGCTTCTCGCGCTGTCCCCGCGTGCAGACTGGTG

-863 GGTGCTGGGCGATGATTAGCTGCAGGGCCCATCCTAGTTTG . . . . . . . . . .

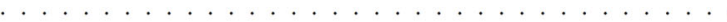
-263................ GCTGTTGTTGTTTGGTTTGGTTTTGTTTTTCAAG SP-1
SP3 AGGGGCCAGGAGAAATGACCCCTTCCCCGCCACGGGTCCCGAAGTGAGGGGGGGGG -203 AGGGGCCAGGAGAAATGACCCTICCCCGCCACGGGTCCCGAAGTGAGGGCGGGGGGGGG TFIID
TFC -83 GGAAGGGGTTAAGGCGCTGAGCGCGGAGCCATCTGGCCGGGTTGGCTGGTTATAACCGCG $-23$

CAGATTCTGTTCACGGGACTCAGAGTTGAAGCTCCTCTCCCTTCCGAACAGCTCCGCGCA 37 CCGCCCCGCGACGCAGCCCGGCGCAACTACTTTCTTCTCTCTCCTTTCTTTCTTCCTCTC 97 СтTTTTCCCCTGCTGGGTAGTGGCTGCGGCGGGGTGGGGAGACTTTGAATGACCGAGCT 157 CGCGTCCACCTTTCTCTTCATGTCGACGTCCCTGGAAACGGCCACACGGATGCCATGGTT 217 ACtTtTGCCACGGTAAGGGGAGGGGC . . . . . . . . . . . . . .

Fig. 2 Identification of the POU3F2 binding site on the NTF3 promoter. a Transcription factor response elements predicted by the Transcription Element Search System for the nucleotide sequence of the NTF3 promoter region $(-1823$ to +243$)$. The transcription start site is indicated as +1 . b Comparison of NTF3 promoter sequence conservation between different species. c Biotin-labeled oligonucleotides containing the intact or mutated POU3F2 binding site were hybridized with total lysates prepared from NT2D1 cells. The POU3F2-DNA complexes were precipitated by streptavidin agarose beads. POU3F2 was analyzed by Western blot analyses. The input of nuclear extracts was used as loading control. Three independent

Immunostaining was visualized following incubation with donkey anti-rabbit-FITC antibody at room temperature for $1 \mathrm{~h}$.

Statistical Analysis All data are presented as mean \pm SEM. The Student's $t$ test or Mann-Whitney $U$ test was used for comparisons between two groups. Comparisons between more than two groups was calculated by one-way ANOVA followed by Bonferroni's post hoc test using GraphPad Prism 5 Software (GraphPad Software, Inc. CA, USA). Results were considered statistically significant at $p<0.05$.

\section{Results}

Induction of NT2D1 Neuronal Differentiation To investigate the expression and regulation of genes in the early stages of neuronal differentiation, we treated NT2D1 cells with
B

Homo sapiens $\quad$ POU3F2 $\quad$ c-Myb

Pan paniscus GTATTTGGATTTTTTGACCTGGGCCCTAACCGAAACACGGAACGGCTTGCTTATTAG

Gorilla Gorilla GTATTTGGATTTTTTGAACCTGGGCCCTAACCGAAACACGGAACGGCTTGCTTATTAG

Pongo abelii GTATTTGGATTTTTGAACCTGGGCCCTAACCGAAACACGGAACGGCTTGCTTATTAG

Mus musculus CTAAAGTACATTGCGAGTTGCTTATCAGACTTTATTAGGAGGCAAGGAAGAGGTGGAC

Rat tus norvegicus GTCAAT--ATTCACTATGTGTATACCATTGCTGCA-CAGGAAACCCTTTCCACCCCAG

Gallus Gallus (-...-.-ATCCAC--AGCAGAAAATATTTCAAAAAATGGTGACAACTATCTAGGTAG
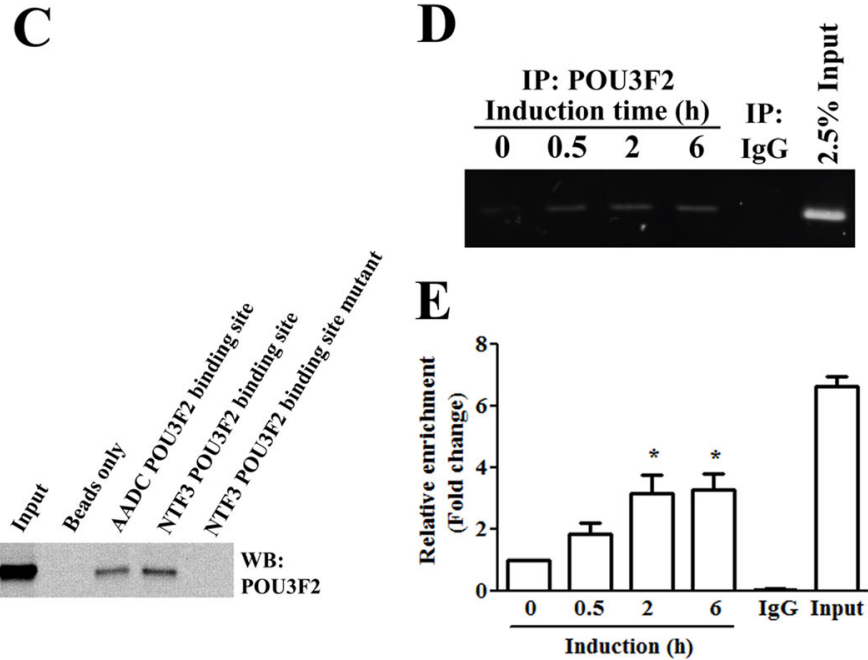

experiments were performed. d Chromatin was prepared from NT2D1 cells treated with induction medium for 0,2 , and $6 \mathrm{~h}$. Cell lysates were mixed with antibodies against POU3F2 or IgG and then precipitated. The precipitates were analyzed by PCR for the presence of the NTF3 promoter sequence. The DNA purified from the sonicated chromatin was directly analyzed by PCR using the ChIP primer, which was used as an input control (Input). e The values of the ChIP DNA were normalized to that of the NT2D1 cells at $0 \mathrm{~h}$ (as a control). Values of fold-change over the control are presented as mean \pm SEM of three independent experiments for d. $* p<0.05$ compared with the control

neuronal induction medium $[23,25,26]$ following the procedure shown in Fig. 1a. Immunofluorescence staining revealed a time-dependent increase in $\beta 3$-tubulin expression, a neuronspecific marker, after exposure to induction medium (Fig. 1b). As shown in Fig. 1c, the percentage of NT2D1 cells positive for $\beta 3$-tubulin staining increased in a time-dependent manner, indicating induction of neuronal differentiation. The degree of $\beta 3$-tubulin expression was consistent with the findings obtained by Western blot analyses (Fig. 1d). In addition, there was a significant time-dependent increase in the expression of POU3F2, but not POU3F3, during neuronal differentiation (Fig. 1d). Interestingly, neuronal induction also increased NTF3 expression (Fig. 1d). Next, we performed nextgeneration-sequencing-based genome-wide POU3F2 binding site screening (ChIP-seq) and bioinformatics-based mapping to identify the potential target genes of POU3F2 during the early neuronal differentiation of NT2D1 cells. A total of 26,742,029 reads were obtained from differentiated NT2D1 
A

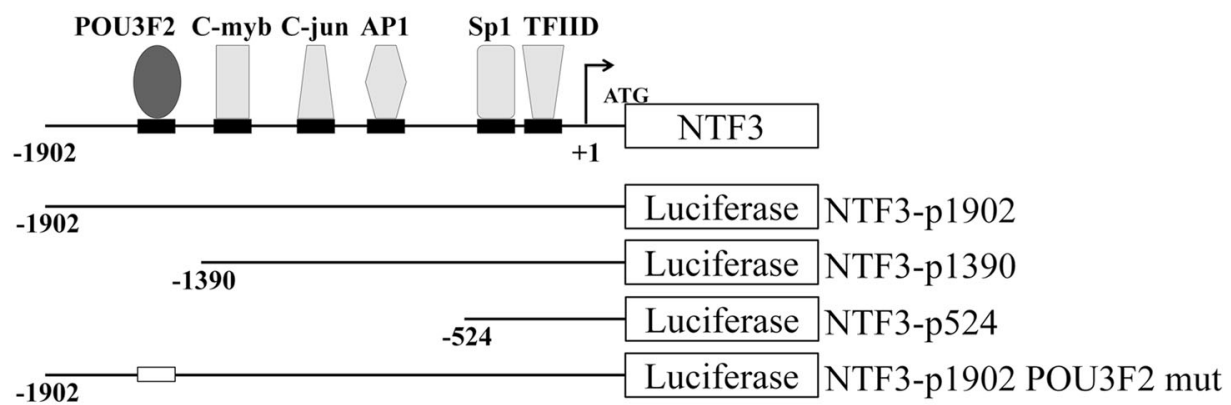

B

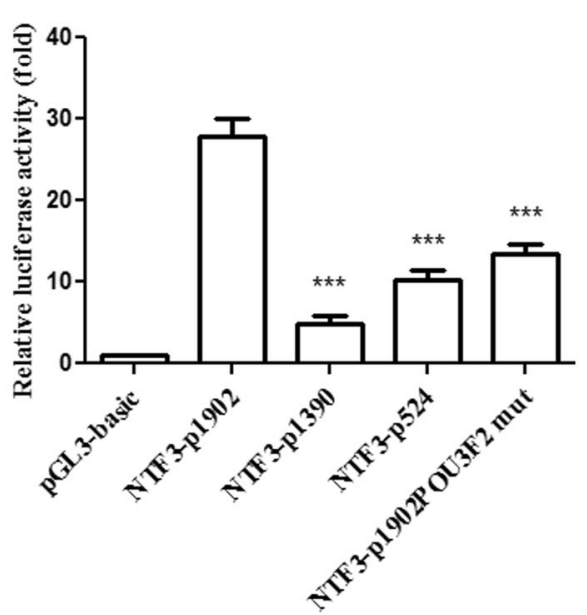

C

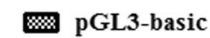 \\ $\infty$ NTF3-p1902 \\ 曰 NTF3-p1902POU3F2 mut
}

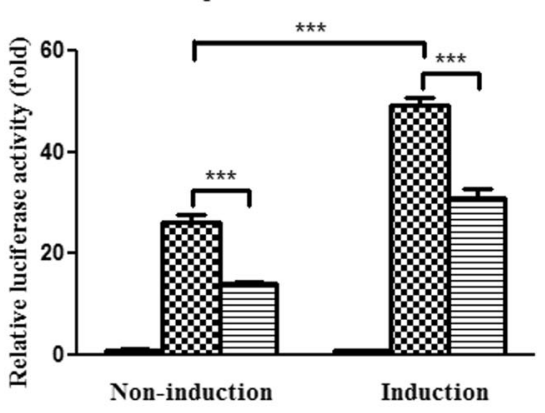

Fig. 3 Effects of POU3F2 on NTF3 promoter activity. a Schematic representation of NTF3-luciferase chimeric constructs. The negative numbers refer to the numbers of bases upstream of the transcription start (+1) site of the NTF3 gene. b NT2D1 cells were transiently transfected with the pGL3 basic vector or NTF3 promoter constructs of different lengths. The luciferase activity of each reporter was normalized to the Renilla luciferase activity and compared with that of cells transfected with the pGL3 basic vector (to which a value of 1 was assigned). $* * * p<0.001$. $\mathrm{c}$ NT2D1 cells were transfected with the pGL3 basic vector, pNTF3-1902, and pNTF3-1902 POU3F2 mut. Approximately $24 \mathrm{~h}$ later, cells were treated with neuronal induction medium. The transcriptional activity of each reporter was normalized to the Renilla luciferase activity and compared with that of cells transfected with the pGL3 basic vector (to which a value of 1 was assigned). $* * * p<0.001$. Values are presented as mean \pm SEM of three independent experiments for $\mathbf{b}$ and $\mathbf{c}$

phosphorylated after neurogenic induction, suggesting that the NTF3/TrkC signaling pathway was activated (Fig. 1g). These results prompted us to examine whether POU3F2 regulates NTF3 expression during neuronal differentiation.

Interaction of POU3F2 with the NTF3 Gene Promoter We further applied bioinformatic tools to analyze the promoter region of the NTF3 gene, an approximately $1.8-\mathrm{kb}$ DNA sequence upstream of the transcription start site, for the prediction of transcriptional regulatory elements. We found a potential POU3F2 response element (nt -1413 to $\mathrm{nt}-$ 1405 ) in the NTF3 promoter (Fig. 2a) using the TESS, suggesting NTF3 as a novel target gene of POU3F2. The flanking sequence of the predicted POU3F2 binding site in the NTF3 promoter was conserved in humans, bonobos, gorillas, and Sumatran orangutans but not in mice, rats, or chickens (Fig. 2b). To confirm the putative binding of POU3F2 to the NTF3 promoter, we performed DAPA in NT2D1 cells. As shown in Fig. 2c, POU3F2 was pulled 
A

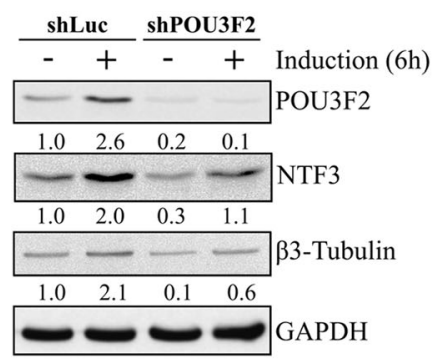

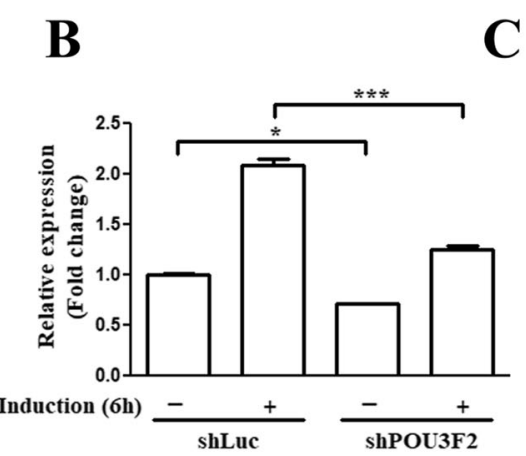

$\mathbf{E}$

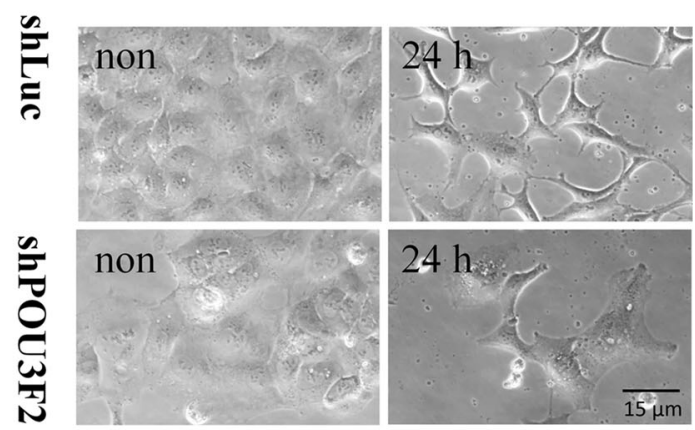

D

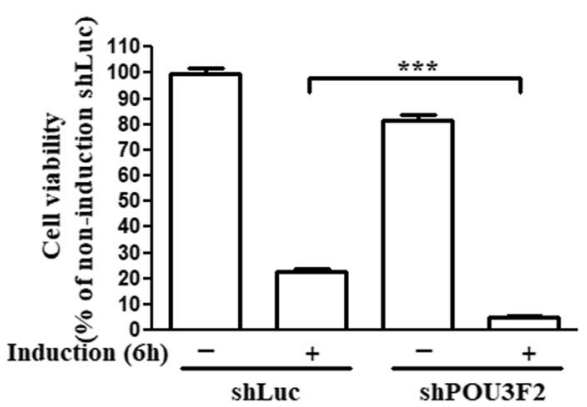

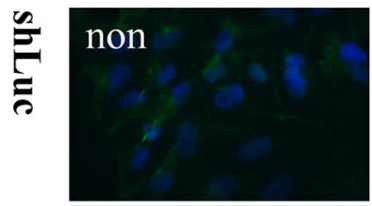

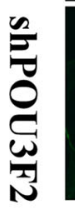

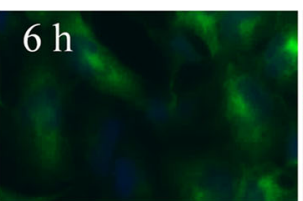

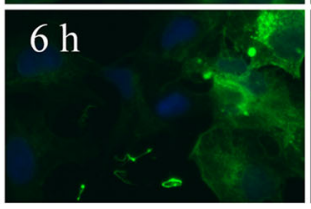

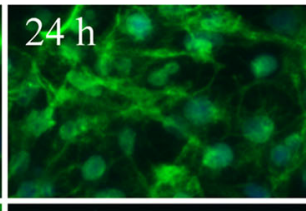

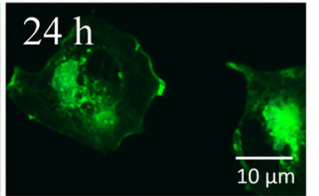

Fig. 4 Effects of POU3F2 silencing on neuronal differentiation and NTF3 expression in NT2D1 cells. a POU3F2 expression in POU3F2knockdown (shPOU3F2) and control (shLuc) NT2D1 cells was determined by Western blot analyses after neuronal induction for $6 \mathrm{~h}$. GAPDH was used as a loading control. The values represent the relative expression compared with that of the non-induced shLuc cells (to which a value of 1 was assigned). $\mathbf{b}$ NTF3 mRNA expression of the cells described in a was analyzed by real-time PCR. mRNA levels were calculated relative to that of the non-induced shLuc cells. ${ }^{*} p<0.05$; $* * * p<0.001$. c Neuronal morphology of shLuc and shPOU3F2 cells

down by the specific probes derived from the promoter of $N T F 3$ and aromatic L-amino acid decarboxylase (AADC, a target gene of POU3F2), but not by the NTF3 probe with the POU3F2 binding site mutated. To investigate the binding ability of POU3F2 to the NTF3 promoter during neuronal differentiation, a ChIP assay was performed in NT2D1 cells exposed to induction medium. The association between POU3F2 and the NTF3 promoter was markedly enhanced after $0.5,2$, and $6 \mathrm{~h}$ of exposure to induction medium, compared with the baseline control (Fig. 2d, e). These results suggested that $\mathrm{POU} 3 \mathrm{~F} 2$ bound to the NTF3 promoter.

POU3F2 Transactivated the Promoter of NTF3 Gene Next, we performed promoter truncation and reporter assays to examine if POU3F2 transactivated the promoter of the NTF3 gene. The luciferase reporter constructs containing regions of the NTF3 promoter varying in length are shown in Fig. 3a. We transfected these constructs individually into NT2D1 cells and found that the luciferase activities of cells receiving NTF3-p1390 and NTF3-p524 were significantly lower than that of cells receiving NTF3-p1902 (Fig. 3b). Moreover, the luciferase activity that were treated with neuronal induction medium for $24 \mathrm{~h}$ or left untreated (non). d Quantification of cell numbers of shLuc and shPOU3F2 described in c. All the percentages of the shLuc and shNTF3 cells were compared to that of the non-induction shLuc cells (to which a value of $100 \%$ was assigned). e $\beta 3$-tubulin staining was performed on shLuc and shPOU3F2 cells, which were treated with neuronal induction medium for 0,6 , or $24 \mathrm{~h}$ or left untreated, after which neuronal cells were detected. Values represent the mean \pm SEM of three independent experiments for $\mathbf{b}$ and $\mathbf{d}$

of cells transfected with the POU3F2 binding sitemutated NTF3-p1902 (designated as NTF3-p1902 POU3F2 mut) was approximately $50 \%$ of that of cells receiving NTF3-p1902 (Fig. 3b). Next, we transfected cells with NTF3-p1902 or NTF3-p1902 POU3F2 mut, and induced cells to undergo neuronal differentiation. As shown in Fig. 3c, the luciferase activity of cells receiving either construct was significantly increased by neuronal induction, compared with non-induction controls. However, an approximately $40 \%$ loss of luciferase activity was observed in the cells receiving NTF3-p1902 POU3F2 mut, compared with that in cells receiving NTF3-p1902. These data demonstrated that the POU3F2 binding element in the NTF3 promoter was important for its activation by neuronal induction and that POU3F2 was able to transactivate NTF3 promoter activity in NT2D1 cells during neuronal differentiation.

POU3F2 Silencing Diminished NTF3 Expression To further address the regulatory role of POU3F2 in NTF3 expression, we knocked down POU3F2 expression in NT2D1 cells with a VSV-G pseudotyped lentivirus-shRNA system 
A

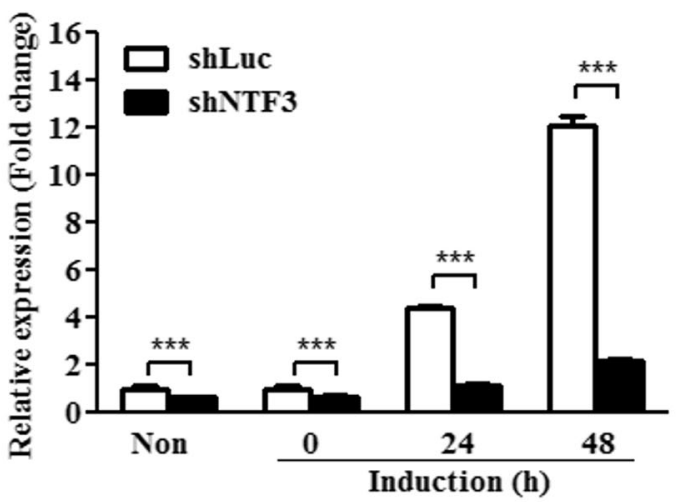

B

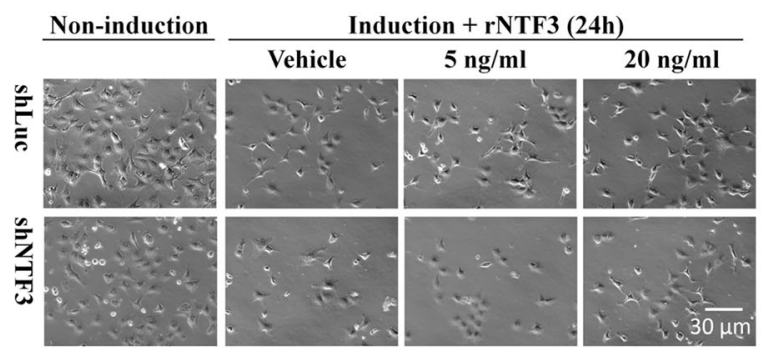

Fig. 5 Effects of NTF3 silencing and NTF3 recombinant protein treatment on the viability and neuronal differentiation of NT2D1 cells. a NTF3 mRNA levels in NTF3-knockdown (shNTF3) and control (shLuc) NT2D1 cells, which were treated with neuronal induction medium for 0,24 , or $48 \mathrm{~h}$ or left untreated (Non), were determined by real-time PCR. mRNA levels were calculated as the relative expression compared with the untreated shLuc cells. ${ }^{* * *} p<0.001$. b Phase contrast microscopy images of untreated shLuc and shNTF3 cells and those cells $24 \mathrm{~h}$ after neuronal induction with concomitant treatment of rNTF3 (5,

and then examined the NTF3 expression. As shown in Fig. 4a, POU3F2 knockdown decreased POU3F2 and NTF3 expression in NT2D1 cells with or without neuronal induction. NTF3 mRNA expression was also significantly inhibited in the POU3F2-knockdown NT2D1 cells, with or without neuronal induction (Fig. 4b). Moreover, we examined the impact of POU3F2 knockdown on neuronal differentiation. We found that the viability of POU3F2knockdown cells was approximately $80 \%$ of that of control cells before neuronal induction and was markedly lowered to approximately one fourth of that of corresponding control cells after neuronal induction for $24 \mathrm{~h}$ (Fig. 4c, d). Immunostaining showed that viable POU3F2-knockdown cells expressed $\beta 3$-tubulin 6 and $24 \mathrm{~h}$ after neuronal induction. However, POU3F2-knockdown cells appeared to diminish the dendritic morphology seen in the control cells (Fig. 4e). These results suggested that POU3F2 played a regulatory role in NTF3 expression and had a critical effect on cell viability during neuronal differentiation.
C

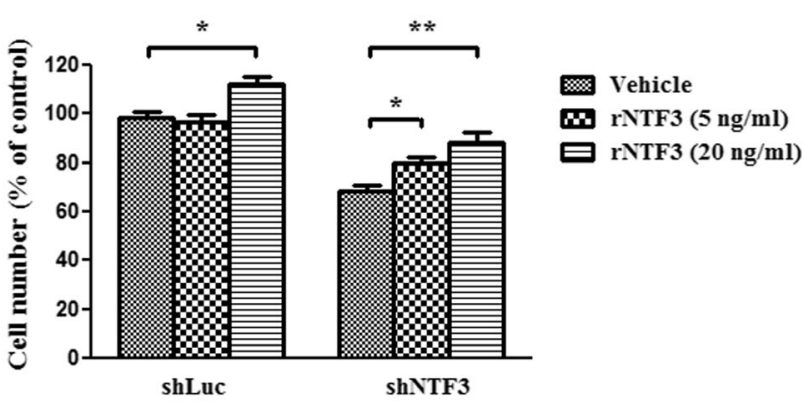

D

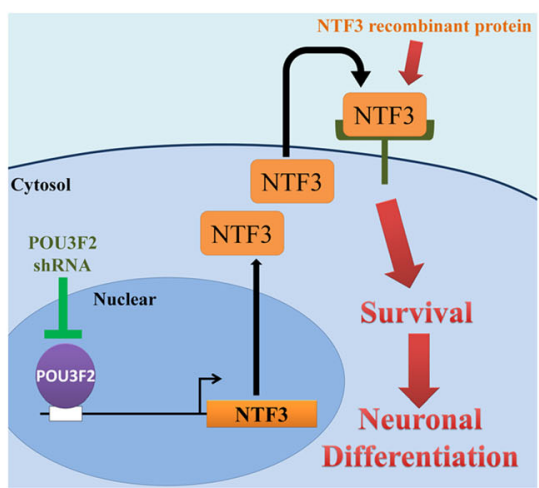

$20 \mathrm{ng} / \mathrm{ml}$ ) or vehicle. c Quantification of neuron number of shLuc and shNTF3 cells as described in $\mathbf{b}$. All the percentages of neurons differentiated from shLuc and shNTF3 cells were compared to that of neurons differentiated from the vehicle-treated shLuc cells (to which a value of $100 \%$ was assigned). $* p<0.05 ; * * p<0.01$. d A suggested model of the POU3F2/NTF3 pathway that mediates the process of neuron differentiation. Values are presented as mean \pm SEM of at least three independent experiments for $\mathbf{a}$ and $\mathbf{c}$

Effects of Recombinant NTF3 Protein on the Neuronal Differentiation of NTF3-Silencing and POU3F2-Silencing NT2D1 Cells Next, to evaluate the role of NTF3 in POU3F2based neuronal differentiation, we knocked down NTF3 expression in NT2D1 cells for subsequent examinations. As shown in Fig. 5a, while neuronal induction increased NTF3 expression time-dependently in control cells, NTF3 knockdown significantly decreased the expression of NTF3 mRNA in the untreated cells and inhibited the induction of NTF3 mRNA expression during neuronal differentiation. After neuronal induction for $24 \mathrm{~h}$, the number of neurons differentiated from the vehicle-treated NTF3-knockdown cells was significantly lowered to $70 \%$ of that from the vehicletreated control cells (Fig. 5b, c). Administration of $5 \mathrm{ng} / \mathrm{ml}$ recombinant NTF3 (rNTF3) did not increase the number of differentiated neurons from control cells but caused an approximately $14.3 \%$ increase in the number of differentiated neurons from NTF3-knockdown cells. At a concentration of $20 \mathrm{ng} / \mathrm{ml}$, rNTF3 caused an approximately 15 and $28.5 \%$ 
Fig. 6 Colocalization of POU3F2 and NTF3 proteins in the developing mouse neurons. a Primary mouse neurons (EN) were prepared from E14.5 embryos, cultured for 7 days, and subjected to double fluorescence immunostaining using anti-NTF3 (green) and anti-POU3F2 (red) antibodies and FITC.

Immunostaining was also performed on cortical sections of mouse brain prepared from E18.5 embryos (b) and postnatal day (P) 0 mice (c). Merged images of NTF3 and POU3F2 showed extensive overlap of expression in primary enriched cultured neurons and in the E18.5 and P0 cortex, mainly at the upper and deeper layers
A
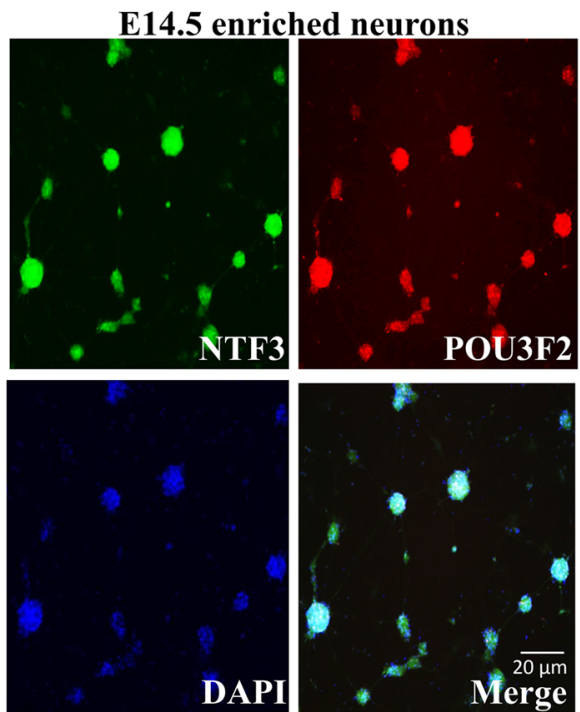

B

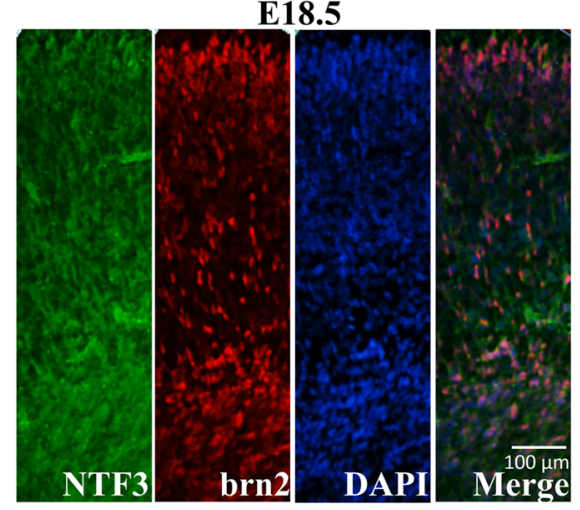

C

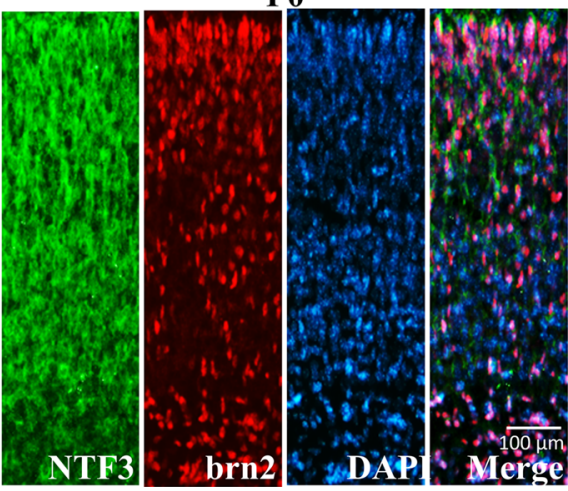

increase in the differentiated neurons from control cells and NTF3-knockdown cells, respectively (Fig. 5c). We also examined the role of NTF3 in the differentiation of POU3F2knockdown cells and found that $20 \mathrm{ng} / \mathrm{ml} \mathrm{rNTF3} \mathrm{significantly}$ increased the number of control and POU3F2-knockdown cells that differentiated into neurons (Supplementary Fig. 1). These results indicated that NTF3 plays an important role in the neuronal differentiation of NT2D1 cells and that the POU3F2/NTF3 pathway is involved in the process of neuron differentiation (Fig. 5d).

POU3F2 and NTF3 Proteins Were Colocalized in the Developing Mouse Neurons In Vitro and In Vivo Given the important role that the POU3F2/NTF3 pathway plays in the neuronal differentiation of human NT2D1 cells, although the flanking sequence of the predicted POU3F2 binding site in human NTF3 promoter is not conserved in mice, rats, and chickens, we thought that this pathway might be also involved in neuronal differentiation in these three species. Therefore, we performed bioinformatic prediction on the $N t f 3$ promoter sequences of these three species and found putative POU3F2 binding sites (mouse: $\mathrm{nt}-1314$ to $\mathrm{nt}-1308$, nt -1206 to nt 1200; rat: $\mathrm{nt}-1110$ to $\mathrm{nt}-1116$, nt -825 to $\mathrm{nt}-831$; chicken: $\mathrm{nt}-814$ to $\mathrm{nt}-808$ ). We subsequently prepared primary enriched neurons from mice at embryonic day (E) 14.5, and brain sections from mice at E18.5 and postnatal day (P) 0 . Double immunostaining using anti-NTF3 (green) and antiPOU3F2 (red) antibodies on the enriched neurons and brain sections showed extensive overlap in the expression of POU3F2 and NTF3 (Fig. 6a-c). These in vivo and in vitro data indicated colocalization of POU3F2 and NTF3 in developing neurons and suggested that the function of POU3F2/ NTF3 pathway in neuronal differentiation is conserved in humans and mice.

\section{Discussion}

POU3F2 expression has been shown to be induced in embryonic stem cell-derived progenitor cells, suggesting that POU3F2 plays an important role in neuronal differentiation [7]. However, little is known about the genes it regulates to mediate neurogenesis. Here, we provide several lines of evidence to show that NTF3 is an important target of POU3F2 in regulating human neuronal differentiation. Our data showed that (i) POU3F2 binds to and transactivates NTF3 in NT2D1 cells with or without neuronal induction (Figs. 2 and 3), (ii) the interaction between POU3F2 and the NTF3 promoter is increased during neuronal differentiation (Fig. 2d), (iii) POU3F2 knockdown decreases NTF3 expression (Fig. 4), and (iv) NTF3 knockdown decreases the number of differentiated neurons, which can be partially recovered by rNTF3 (Fig. 5). These results depicted a regulatory role of POU3F2 in NTF3 expression and suggested that POU3F2 might maintain the basal expression of NTF3 in neuroprogenitor cells and 
further increase NTF3 expression in response to neuronal induction.

In fact, given our finding that rNTF3 $(20 \mathrm{ng} / \mathrm{ml})$ increased the number of differentiated neurons from POU3F2knockdown cells (Supplementary Fig. 1), NTF3 could be an important downstream mediator of POU3F2 in promoting human neuronal differentiation. It is worthy of note that the outgrowth dendrite of neurons differentiated from POU3F2knockdown NT2D1 cells was significantly abolished compared with those from control cells (Fig. 4c, e). These results indicate that POU3F2 also plays a role in the development of neuronal morphology.

On the other hand, although the flanking sequence of the predicted POU3F2 binding site in the human NTF3 promoter was not conserved in mice, rats, and chickens (Fig. 2b), bioinformatics prediction showed the existence of putative POU3F2 binding sites in the $N t f 3$ promoter sequences of these three species. Indeed, our data demonstrated the colocalization and coexpression of POU3F2 and NTF3 in mouse primary neuron cultures and in the brain tissues of mice at E18.5 and P0 (Fig. 6), which also supported a regulatory role of the POU3F2/NTF3 pathway in mouse neuronal development.

Our results showed that the promoter activity of NTF3p1390 and NTF3-p1902 POU3F2 mut were approximately 80 and 50\% lower, respectively, than that of NTF3-p1902 (Fig. 3), indicating that the protein-DNA interactions taking place in the region from nt -1902 to $n t-1930$ of the NTF3 promoter were important contributors to its transactivation. Using transcription factor prediction software, we found a putative SRY response element and several putative Spfamily response elements in the human NTF3 promoter ranging from $n t-1920$ to $n t-1390$. It has been reported that Spfamily transcription factors and SRY positively regulate the Ntf3 promoter in mouse neuron and rat testis, respectively $[29,30]$. Furthermore, whereas the promoter activity of NTF3-p524 was approximately 50\% higher than that of NTF3-p1390 (Fig. 3), sequence analysis of the NTF3 promoter region ranging from $n t-1390$ to $n t-524$ also revealed several putative binding sites for some important differentiation-related transcription factors such as CTCF, YY1, GATA-3, and Sp-1 [31]. In addition, the regulatory role of Zic 1 in neuronal differentiation has been addressed in embryonic mouse models [16]. We found that POU3F2 knockdown resulted in decreased Zic1 expression in NT2D1 cells with or without neuronal induction (Supplementary Fig. 2). We also found a putative Zic1 binding site in the human NTF3 promoter via bioinformatics prediction. Therefore, POU3F2 may also regulate NTF3 expression via the POU3F2/Zic 1 axis in developing human neurons. While our data, taken together, depict POU3F2 as a transactivator of the NTF3 gene during neuronal differentiation, it is possible that POU3F2 may target other factors for NTF3 induction and that transcription factors other than POU3F2 may be also involved in NTF3 induction. More studies are needed to clarify the transcriptional network which regulates NTF3 promoter in response to neurogenic induction.

To the best of our knowledge, this is the first study to reveal a novel function of POU3F2 in regulating the promoter activity and expression of NTF3. Furthermore, we found that NTF3 promotes the survival/differentiation cascade during neuronal differentiation. Our findings provide further evidence that human neuronal differentiation and survival is partially governed by the POU3F2/NTF3 pathway.

Acknowledgements We thank Chih-Hao Wu for excellent technical assistance.

Funding Information This study was supported in part by grants NSC100-2314-B-371-004 and NSC 101-2314-B-371-006-MY3 from the National Science Council, Taiwan.

\section{Compliance with Ethical Standards}

Conflict of Interest The authors report no conflict of interest.

Open Access This article is distributed under the terms of the Creative Commons Attribution 4.0 International License (http:// creativecommons.org/licenses/by/4.0/), which permits unrestricted use, distribution, and reproduction in any medium, provided you give appropriate credit to the original author(s) and the source, provide a link to the Creative Commons license, and indicate if changes were made.

\section{References}

1. Spaniol P, Bornmann C, Hauptmann G, Gerster T (1996) Class III POU genes of zebrafish are predominantly expressed in the central nervous system. Nucleic Acids Res 24(24):4874-4881

2. Nakai S, Kawano H, Yudate T, Nishi M, Kuno J, Nagata A, Jishage $\mathrm{K}$, Hamada $\mathrm{H}$ et al (1995) The POU domain transcription factor Brn-2 is required for the determination of specific neuronal lineages in the hypothalamus of the mouse. Genes Dev 9(24):3109-3121

3. Cook AL, Sturm RA (2008) POU domain transcription factors: BRN2 as a regulator of melanocytic growth and tumourigenesis. Pigment Cell Melanoma Res 21(6):611-626. https://doi.org/10. 1111/j.1755-148X.2008.00510.x

4. Schreiber J, Enderich J, Sock E, Schmidt C, Richter-Landsberg C, Wegner M (1997) Redundancy of class III POU proteins in the oligodendrocyte lineage. J Biol Chem 272(51):32286-32293

5. Aiba K, Sharov AA, Carter MG, Foroni C, Vescovi AL, Ko MS (2006) Defining a developmental path to neural fate by global expression profiling of mouse embryonic stem cells and adult neural stem/progenitor cells. Stem Cells 24(4):889-895. https://doi.org/ 10.1634/stemcells.2005-0332

6. Fujii H, Hamada H (1993) A CNS-specific POU transcription factor, Brn-2, is required for establishing mammalian neural cell lineages. Neuron 11(6):1197-1206

7. Hauptmann G, Gerster T (2000) Combinatorial expression of zebrafish Brn-1- and Brn-2-related POU genes in the embryonic brain, pronephric primordium, and pharyngeal arches. Dev Dyn: Off Publ Am Assoc Anatomists 218(2):345-358. https://doi.org/ 10.1002/(SICI)1097-0177(200006)218:2<345::AID-DVDY8>3.0. $\mathrm{CO} ; 2-\mathrm{V}$ 
8. McEvilly RJ, de Diaz MO, Schonemann MD, Hooshmand F, Rosenfeld MG (2002) Transcriptional regulation of cortical neuron migration by POU domain factors. Science 295(5559):1528-1532. https://doi.org/10.1126/science.1067132

9. Sugitani M, Sugai T, Onoda N (2002) Postsynaptic activity of metabotropic glutamate receptors in the piriform cortex. Neuroreport 13(11):1473-1476

10. Dominguez MH, Ayoub AE, Rakic P (2013) POU-III transcription factors (Brn1, Brn2, and Oct6) influence neurogenesis, molecular identity, and migratory destination of upper-layer cells of the cerebral cortex. Cereb Cortex 23(11):2632-2643. https://doi.org/10. 1093/cercor/bhs252

11. Pang ZP, Yang N, Vierbuchen T, Ostermeier A, Fuentes DR, Yang TQ, Citri A, Sebastiano V et al (2011) Induction of human neuronal cells by defined transcription factors. Nature 476(7359):220-223. https://doi.org/10.1038/nature10202

12. Vierbuchen T, Wernig M (2011) Direct lineage conversions: unnatural but useful? Nat Biotechnol 29(10):892-907. https://doi.org/10. 1038/nbt. 1946

13. Zhou Z, Kohda K, Ibata K, Kohyama J, Akamatsu W, Yuzaki M, Okano HJ, Sasaki E et al (2014) Reprogramming non-human primate somatic cells into functional neuronal cells by defined factors. Mol Brain 7:24. https://doi.org/10.1186/1756-6606-7-24

14. Vierbuchen T, Ostermeier A, Pang ZP, Kokubu Y, Sudhof TC, Wernig M (2010) Direct conversion of fibroblasts to functional neurons by defined factors. Nature 463(7284):1035-1041. https:// doi.org/10.1038/nature08797

15. Lin M, Pedrosa E, Shah A, Hrabovsky A, Maqbool S, Zheng D, Lachman HM (2011) RNA-Seq of human neurons derived from iPS cells reveals candidate long non-coding RNAs involved in neurogenesis and neuropsychiatric disorders. PLoS One 6(9): e23356. https://doi.org/10.1371/journal.pone.0023356

16. Urban S, Kobi D, Ennen M, Langer D, Le Gras S, Ye T, Davidson I (2015) A Brn2-Zic1 axis specifies the neuronal fate of retinoic-acidtreated embryonic stem cells. J Cell Sci 128(13):2303-2318. https:// doi.org/10.1242/jes. 168849

17. Hamatake M, Miyazaki N, Sudo K, Matsuda M, Sadakata T, Furuya A, Ichisaka S, Hata Y et al (2011) Phase advance of the light-dark cycle perturbs diurnal rhythms of brain-derived neurotrophic factor and neurotrophin-3 protein levels, which reduces synaptophysin-positive presynaptic terminals in the cortex of juvenile rats. J Biol Chem 286(24):21478-21487. https://doi.org/10. 1074/jbc.M110.195859

18. Wang Q, Green SH (2011) Functional role of neurotrophin-3 in synapse regeneration by spiral ganglion neurons on inner hair cells after excitotoxic trauma in vitro. J Neurosci: Off J Soc Neurosci 31(21):7938-7949. https://doi.org/10.1523/JNEUROSCI.1434-10. 2011

19. Chao MV (2003) Neurotrophins and their receptors: a convergence point for many signalling pathways. Nat Rev Neurosci 4(4):299 309. https://doi.org/10.1038/nrn1078

20. Liot G, Gabriel C, Cacquevel M, Ali C, MacKenzie ET, Buisson A, Vivien D (2004) Neurotrophin-3-induced PI-3 kinase/Akt signaling rescues cortical neurons from apoptosis. Exp Neurol 187(1):38-46. https://doi.org/10.1016/j.expneurol.2004.01.002

21. Conover JC, Yancopoulos GD (1997) Neurotrophin regulation of the developing nervous system: analyses of knockout mice. Rev Neurosci 8(1):13-27

22. Usui N, Watanabe K, Ono K, Tomita K, Tamamaki N, Ikenaka K, Takebayashi H (2012) Role of motoneuron-derived neurotrophin 3 in survival and axonal projection of sensory neurons during neural circuit formation. Development 139(6):1125-1132. https://doi.org/ 10.1242/dev.069997

23. Goodfellow CE, Graham SE, Dragunow M, Glass M (2011) Characterization of NTera2/D1 cells as a model system for the investigation of cannabinoidfunction in human neurons and astrocytes. J Neurosci Res 89(10):1685-1697. https://doi.org/10.1002/ jnr.22692

24. Wu HM, Tzeng NS, Qian L, Wei SJ, Hu X, Chen SH, Rawls SM, Flood P et al (2009) Novel neuroprotective mechanisms of memantine: increase in neurotrophic factor release from astroglia and anti-inflammation by preventing microglial activation. Neuropsychopharmacology 34(10):2344-2357. https://doi.org/10. 1038/npp. 2009.64

25. Hung SC, Cheng H, Pan CY, Tsai MJ, Kao LS, Ma HL (2002) In vitro differentiation of size-sieved stem cells into electrically active neural cells. Stem Cells 20(6):522-529. https://doi.org/10. 1634/stemcells.20-6-522

26. Romero-Ramos M, Vourc'h P, Young HE, Lucas PA, Wu Y, Chivatakarn O, Zaman R, Dunkelman N, el-Kalay MA, Chesselet MF (2002) Neuronal differentiation of stem cells isolated from adult muscle. J Neurosci Res 69 (6):894-907. doi:https://doi.org/ $10.1002 /$ jnr. 10374

27. Hsin IL, Ou CC, Wu MF, Jan MS, Hsiao YM, Lin CH, Ko JL (2015) GMI, an immunomodulatory protein from Ganoderma microsporum, potentiates cisplatin-induced apoptosis via autophagy in lung cancer cells. Mol Pharm 12(5):1534-1543. https://doi. org/10.1021/mp500840z

28. Hsin IL, Wang SC, Li JR, Ciou TC, Wu CH, Wu HM, Ko JL (2016) Immunomodulatory proteins FIP-gts and chloroquine induce caspase-independent cell death via autophagy for resensitizing cisplatin-resistant urothelial cancer cells. Phytomedicine: Int J Phytother Phytopharmacol 23(13):1566-1573. https://doi.org/10. 1016/j.phymed.2016.09.003

29. Ishimaru N, Tabuchi A, Hara D, Hayashi H, Sugimoto T, Yasuhara M, Shiota J, Tsuda M (2007) Regulation of neurotrophin-3 gene transcription by Sp3 and Sp4 in neurons. J Neurochem 100(2):520 531. https://doi.org/10.1111/j.1471-4159.2006.04216.x

30. Clement TM, Bhandari RK, Sadler-Riggleman I, Skinner MK (2011) SRY directly regulates the neurotrophin 3 promoter during male sex determination and testis development in rats. Biol Reprod 85(2):277-284. https://doi.org/10.1095/biolreprod.110.090282

31. Weirauch MT, Yang A, Albu M, Cote AG, Montenegro-Montero A, Drewe P, Najafabadi HS, Lambert SA et al (2014) Determination and inference of eukaryotic transcription factor sequence specificity. Cell 158(6):1431-1443. https://doi.org/10.1016/j.cell.2014.08. 009 